\title{
Area-Preserving Diffeomorphisms and Supermembrane Lorentz Invariance
}

\author{
B. de Wit ${ }^{1}$, U. Marquard ${ }^{2}$ and H. Nicolai ${ }^{3}$ \\ ${ }^{1}$ Institute for Theoretical Physics, University of Utrecht, Princetonplein 5, NL-3584 CC Utrecht, \\ The Netherlands \\ 2 Deutsches Elektronen-Synchroton, DESY, Notkestrasse 85, D-2000 Hamburg 52, FRG \\ ${ }^{3}$ II. Institute for Theoretical Physics, University of Hamburg, Luruper Chaussee 149, D-2000 Hamburg \\ 50, FRG
}

\begin{abstract}
The Lie algebra of area-preserving diffeomorphisms on closed membranes of arbitrary topology is investigated. On the basis of a harmonic decomposition we define the structure constants as well as two other tensors which appear in the supermembrane Lorentz generators. We derive certain identities between these tensors and analyze their validity when the areapreserving diffeomorphisms are approximated by $S U(N)$. One of the additional tensors can then be identified with the invariant symmetric three-index tensor of $S U(N)$, while the second has no obvious analog. We prove that the Lorentz generators are classically conserved in the light-cone gauge for arbitrary membrane topology, as a consequence of these tensor identities. This formulation allows a systematic study of the violations of Lorentz invariance in the $S U(N)$ approximation.
\end{abstract}

\section{Introduction}

The recent interest in (super)membrane theory has been motivated as much by their mathematical structure as by their possible interest for the unification of fundamental interactions (recent developments are reviewed in [1]). Of particular importance is the group of area-preserving (symplectic) diffeomorphisms which naturally appears in membrane theory as a residual symmetry in the light-cone gauge $[2,3]$ (the corresponding symmetry in string theory consists only of the "length-preserving" diffeomorphism $\sigma \rightarrow \sigma+$ constant, and is thus rather trivial). Little is known about these infinite-dimensional groups in general (for an early reference in the physics literature, see [4]), and one of the possible benefits of membrane theories could well be an improved understanding of their mathematical structure. A remarkable result, already obtained some time ago, is that, for spherical membranes, the structure constants of the group of area-preserving diffeomorphisms can be obtained as the $N \rightarrow \infty$ limit of the $S U(N)$ structure constants $[2,3]$. More recently, and partly motivated by the discovery of supermembranes [5], several papers have appeared dealing with area-preserving diffeomorphisms on the sphere and the torus [6-12], which have led to a certain amount of progress. 
For instance, it was realized that (the simple subgroup of) the area-preserving diffeomorphisms on the torus can also be obtained from $S U(\infty)([10,12,19]$ and Appendix A of this paper); this result, however, does not necessarily imply that the area-preserving diffeomorphisms for spherical and toroidal membranes are the "same." As for topologically more complicated membranes, results are scarce due to the lack of explicit representations for the associated Lie algebras. Another complication is that the Lie algebra associated with the group of area-preserving diffeomorphisms is no longer simple for nonzero genus (it is simple for the sphere [13]). This follows from the observation that, for nonzero genus, there are diffeomorphisms generated by harmonic vector fields, such that the remaining area-preserving diffeomorphisms constitute an invariant subgroup.

The group of area-preserving diffeomorphisms is also important for another reason. It was shown in [7] that supermembranes can be formulated as onedimensional supersymmetric gauge theories of area-preserving diffeomorphisms. The supermembrane Hamiltonian can thus be regarded as a one-dimensional reduction of $N=4$ super-Yang-Mills theory with an infinite-dimensional gaugegroup. A supersymmetric regularization of this theory is obtained by approximating the group of area-preserving diffeomorphisms by a finite-dimensional gauge group. In this way, the supermembrane can be analyzed as a system in supersymmetric quantum mechanics. Clearly, a regularization is not only useful but, in fact, necessary if one is to gain any understanding of the nonperturbative dynamics of supermembranes, just as it is necessary for any other interacting (and possibly divergent) field theory. For instance, by employing such a regularization it was demonstrated recently that the spectrum of the associated supersymmetric Hamiltonian is continuous and has no gap [14]. We repeat, however, that the validity of this result is contingent upon the approximability of the group of area-preserving transformations by a finite Lie group.

In Sect. 2 of this paper we will give a systematic treatment of area-preserving transformations (for related discussions, see e.g. [8]). The presentation is set up in a way suited to the treatment of the (classical) Lorentz invariance in the light-cone gauge, which will follow in Sect. 3. Performing a harmonic expansion of all dynamically relevant variables in a basis of scalar harmonics on the membrane, one is naturally led to define the structure constants $f_{A B C}$ of the area-preserving transformations in terms of a certain overlap integral involving these harmonics $[2,3]$. Our analysis of the Lorentz generators reveals the existence of two further tensors, which to the best of our knowledge have not appeared in the literature so far. In the $S U(N)$ approximation, one of these, denoted by $d_{A B C}$, corresponds to the $S U(N)$ - invariant symmetric three-index tensor; the other, denoted by $c_{A B C}$, has no $S U(N)$ analog. The existence of such a tensor $c_{A B C}$ different from $f_{A B C}$ and $d_{A B C}$ is a genuine property of the infinite-dimensional group that distinguishes it from its finite-dimensional "regulator" subgroups. Namely, we will show explicitly that a tensor with analogous properties can always be redefined to become a linear combination of the known group invariant tensors if the Lie algebra possesses a Killing-Cartan metric (for the infinite-dimensional group of area-preserving diffeomorphisms this metric is singular). Relying on the completeness relations for the scalar and vector harmonics on the membrane, we deduce a number of identities 
for these tensors. Using these identities it is thus possible to establish the light-cone Lorentz invariance in an "algebraic" fashion. One of the advantages of the present formulation is that we can treat arbitrary membrane topologies. Furthermore, it is now easy to study the violations of Lorentz invariance that appear in the $S U(N)$ approximation. Not unexpectedly, we find that the boost generators are only conserved modulo terms of order $O\left(1 / N^{2}\right)$, but these terms can be controlled and shown to vanish in the $N \rightarrow \infty$ limit. In two appendices we describe how the torus and the sphere algebra can be approximated by $S U(N)$ and give explicit formulae for all the tensors that were introduced before.

Having established the classical Lorentz invariance in the large- $N$ limit, it follows that the Lorentz algebra must close in the usual fashion in this limit. One might wonder whether the formalism is also useful in order to study the closure of the quantum Lorentz algebra and thus could lead to the determination of the critical dimension of the supermembrane. Indeed, treating the variables as quantum operators and paying attention to the order in which they appear one would pick up extra contributions which stay finite or diverge in the limit $N \rightarrow \infty$. However, their determination would not be sufficient for the following reason. Before embarking on such a calculation one would have to ensure first that the Lorentz generators are well-defined as quantum operators. In string theory, this is achieved by normal ordering, which is sufficient to render all operators well-defined, because string theory is essentially free. In contrast, the (super)membrane is described as a three-dimensional interacting quantum field theory. Therefore one cannot a priori expect the operators to become well-defined through normal ordering alone. Rather, one must treat them as composite operators in interacting field theory with all the concomitant complications. This, in turn, requires the order-by-order calculation of the relevant renormalizations and counterterms. As to date no such results are available, it does not make much sense to further pursue the question of the quantum Lorentz algebra at this point. To be sure, one can attempt an analysis of the short distance singularities based on certain plausible "kinematical" assumptions about the operator product expansions [15]. It is indeed possible with rather mild assumptions to predict the space-time dimension $D=27$ as a necessary condition for the consistency of the bosonic membrane [15] (the same procedure yields $D=11$ for the supermembrane [16]). In conclusion, there is some evidence of an underlying mathematical structure leading beyond string theory, but much more work is obviously needed to explain the "enigma" of $D=11$ supergravity.

\section{Area-Preserving Diffeomorphisms and Harmonic Decompositions}

In this paper we will be considering closed membranes, so that the membrane coordinates and momenta can be expressed as functions of two parameters, $\sigma^{1}$ and $\sigma^{2}$, which are local coordinates on a closed compact two-dimensional space of the same topology as the membrane. In this parameter space it is convenient to define a metric, which we shall denote by $w_{r s}(\sigma)$ to avoid confusion with the (dynamically relevant) Nambu-Goto metric of the membrane which we denote by $g_{r s}$. Without loss of generality we may fix the total area of the parameter space to 
unity, ${ }^{1}$ i.e.,

$$
\int d^{2} \sigma \sqrt{w(\sigma)}=1
$$

Area-preserving diffeomorphisms are diffeomorphisms

$$
\sigma^{r} \rightarrow \sigma^{r}+\xi^{r}(\sigma)
$$

with

$$
\partial_{r}\left(\sqrt{w(\sigma)} \xi^{r}(\sigma)\right)=0, \quad \text { or, equivalently, } \quad D_{r} \xi^{r}(\sigma)=0,
$$

where $w(\sigma)=\operatorname{det} w_{r s}(\sigma)$ and $D_{r}$ is the covariant derivative with respect to the metric $w_{r s}$. The general solution of (2.3) can be decomposed into co-exact and harmonic vector fields. Furthermore, there can be homotopically nontrivial transformations; these will not be considered in what follows. The co-exact components can be parametrized in terms of globally defined functions $\xi(\sigma)$,

$$
\xi^{r}(\sigma)=\frac{\varepsilon^{r s}}{\sqrt{w(\sigma)}} \partial_{s} \xi(\sigma) .
$$

Under these diffeomorphisms, the (transverse) membrane coordinates $\vec{X}$ transform as

$$
\delta X^{a}=\left\{\xi, X^{a}\right\}
$$

where the bracket $\{A, B\}$ for two functions $A(\sigma)$ and $B(\sigma)$ is defined by

$$
\{A, B\}(\sigma) \equiv \frac{1}{\sqrt{w(\sigma)}} \varepsilon^{r s} \partial_{r} A(\sigma) \partial_{s} B(\sigma) .
$$

This bracket can be shown to have the requisite properties of a Lie bracket. In particular it satisfies the Jacobi identity,

$$
\{A,\{B, C\}\}+\{B,\{C, A\}\}+\{C,\{A, B\}\}=0 .
$$

The harmonic component of area-preserving diffeomorphisms can be decomposed into a set of harmonic vectors, denoted by $\Phi_{r}^{(\lambda)}$, which satisfy

$$
D_{r} \Phi^{(\lambda) r}=D_{[r} \Phi_{s]}^{(\lambda)}=0 .
$$

For a membrane of genus $g$ there are precisely $2 g$ independent harmonic vectors.

It is clear that the diffeomorphisms generated by the co-exact vector fields form a group; this group will be denoted by $G$. The commutator of two infinitesimal $G$-transformations characterized by functions $\xi_{1}$ and $\xi_{2}$ yields a similar transformation characterized by

$$
\xi_{3}=\left\{\xi_{2}, \xi_{1}\right\}
$$

One can also include the transformations generated by harmonic vector fields. The commutator of such an infinitesimal transformation generated by $\Phi^{(\lambda) r}$ with a $G$-transformation characterized by $\xi_{1}$ is again a $G$-transformation characterized by

$$
\xi_{2}=\Phi^{(\lambda) r} \partial_{r} \xi_{1}
$$

\footnotetext{
1 Note that in previous publications (e.g. [7]) we used $w$ rather than $\sqrt{w}$
} 
Furthermore, the commutator of two infinitesimal transformations generated by $\Phi^{(\lambda) r}$ and $\Phi^{\left(\lambda^{\prime}\right) r}$ yields also a $G$-transformation characterized by

$$
\xi=\sqrt{w(\sigma)} \varepsilon_{r s} \Phi^{(\lambda) r} \Phi^{\left(\lambda^{\prime}\right) s} .
$$

The above commutation relations show that $G$ is an invariant subgroup of the full group of (homotopically trivial) area-preserving diffeomorphisms. Hence the latter group is in general not simple. Observe that this remark is not relevant for spherical membranes, where no harmonic vectors exist, while for toroidal membranes (2.11) is constant so that the commutator vanishes. However, in most of this paper we will be concerned with the subgroup $G$. As we shall discuss below, it is possible to view this group as the $N \rightarrow \infty$ limit of $S U(N)$, at least for spherical and toroidal membranes.

To exhibit the group of area-preserving diffeomorphisms in a convenient fashion one expands the membrane coordinates into a complete orthonormal basis of functions $Y^{I}(\sigma)$ (where $I=0,1, \ldots$ ) according to

$$
\vec{X}(\sigma)=\sum_{I} \vec{X}^{I} Y_{I}(\sigma),
$$

and likewise for all other quantities of interest such as momenta or fermionic coordinates. The basis functions $Y_{I}$, which can be chosen real or complex, are normalized according to

where

$$
\int d^{2} \sigma \sqrt{w(\sigma)} Y^{I}(\sigma) Y_{J}(\sigma)=\delta_{J}^{I},
$$

$$
Y^{I} \equiv Y_{I}^{*}=\eta^{I J} Y_{J} .
$$

The last equation follows from the completeness of the functions $Y_{I}$. The tensor $\eta_{I J}$ is thus given by

$$
\eta_{I J} \equiv \int d^{2} \sigma \sqrt{w(\sigma)} Y_{I}(\sigma) Y_{J}(\sigma) .
$$

It is now convenient to split the basis functions into the constant mode $Y_{0}=1$, which characterizes the center-of-mass coordinate of the membrane, i.e.,

$$
\vec{X}_{0}=\int d^{2} \sigma \sqrt{w(\sigma)} \vec{X}(\sigma),
$$

and the remaining functions $Y_{A}$, where here and throughout the text the indices $A$ will run over the positive integers. The completeness of the basis functions now implies

$$
\sum_{A} Y^{A}(\sigma) Y_{A}(\rho)=\frac{1}{\sqrt{w(\sigma)}} \delta^{(2)}(\sigma, \rho)-1,
$$

where the last term is due to the fact that the constant function $Y_{0}$ is not included on the left-hand side. Observe that we made use of the normalization (2.1).

For the basis functions $Y_{I}$ one may choose the eigenfunctions of the covariant Laplacian $\Delta=D^{r} D_{r}$, so that

$$
\Delta Y_{0}=0, \quad \Delta Y_{A}=-\omega_{A} Y_{A},
$$

where $\omega_{A}>0$. The Green's function of the Laplacian can then be expressed in 
terms of this basis, viz.

$$
G(\sigma, \rho)=-\sum_{A} \frac{1}{\omega^{A}} Y^{A}(\sigma) Y_{A}(\rho)
$$

so that

$$
\Delta G(\sigma, \rho)=\frac{1}{\sqrt{w(\sigma)}} \delta^{(2)}(\sigma, \rho)-1 .
$$

Below we will also need another Green's function in order to solve the equation

$$
\partial_{r} X^{-}=V_{r},
$$

where $X^{-}$is a light-cone coordinate of the membrane, while $V_{r}$ is defined in terms of the transverse coordinates and momenta as well as fermionic coordinates. As $X^{-}$must be a single-valued function of $\sigma$ the consistency of (2.21) requires that the vector field $V^{r}$ satisfies the following equations:

$$
D_{[r} V_{s]}=0, \int d^{2} \sigma \sqrt{w(\sigma)} \Phi^{(\lambda) r}(\sigma) V_{r}(\sigma)=0 .
$$

Both these equations are equivalent to requiring that $V_{r}$ vanishes when integrated along any closed loop, i.e.,

$$
\oint \sqrt{w(\sigma)} V_{r}(\sigma) d \sigma^{r}=0
$$

To express the solution of (2.21) in terms of $V_{r}$, let us first discuss the decomposition of vector fields in terms of "vector harmonics." From the Hodge decomposition, it follows that a basis for them is given by

$$
Y_{r}^{(1) A}(\sigma)=\frac{1}{\sqrt{\omega^{A}}} \partial_{r} Y^{A}(\sigma), \quad Y_{r}^{(2) A}(\sigma)=\frac{1}{\sqrt{\omega^{A}}} \sqrt{w(\sigma)} \varepsilon_{r s} \partial^{s} Y^{A}(\sigma)
$$

and the harmonic vectors $\Phi_{r}^{(\lambda)}$. These vector fields are eigenfunctions of the Lichnerowicz operator, which, in two dimensions, reads

$$
\Delta_{L}=D^{s} D_{s}+\frac{1}{2} R,
$$

where $R$ is the scalar curvature. The eigenvalues of $Y_{r}^{(1) A}, Y_{r}^{(2) A}$ and $\Phi_{r}^{(\lambda)}$ are given by

$$
\Delta_{L} Y_{r}^{(\alpha) A}(\sigma)=-\omega_{A} Y_{r}^{(\alpha) A}(\sigma), \quad \Delta_{L} \Phi_{r}^{(\lambda)}=0 \quad(\alpha=1,2) .
$$

With a suitable choice for the harmonic vectors the functions $Y_{r}^{(1) A}, Y_{r}^{(2) A}$ and $\Phi_{r}^{(2)}$ are orthonormal,

$$
\begin{gathered}
\int d^{2} \sigma \sqrt{w(\sigma)} w^{r s}(\sigma) Y_{r}^{(\alpha) A}(\sigma) Y_{s}^{(\beta) B}(\sigma)=\delta^{\alpha \beta} \eta^{A B} \\
\int d^{2} \sigma \sqrt{w(\sigma)} w^{r s}(\sigma) Y_{r}^{(\alpha) A}(\sigma) \Phi_{s}^{(\lambda)}(\sigma)=0 \\
\int d^{2} \sigma \sqrt{w(\sigma)} w^{r s}(\sigma) \Phi_{r}^{(\lambda)}(\sigma) \Phi_{s}^{\left(\lambda^{\prime}\right)}(\sigma)=\delta^{\lambda \lambda^{\prime}}
\end{gathered}
$$

The corresponding completeness relation reads

$$
\sum_{A, \alpha} Y_{r}^{(\alpha) A}(\sigma) Y_{s A}^{(\alpha)}(\rho)=\frac{w_{r s}(\sigma)}{\sqrt{w(\sigma)}} \delta^{(2)}(\sigma, \rho)-\sum_{\lambda} \Phi_{r}^{(\lambda)}(\sigma) \Phi_{s}^{(\lambda)}(\rho) .
$$


With the above results it is not difficult to establish that the solution of (2.21) is

$$
X^{-}(\sigma)=\int d^{2} \rho \sqrt{w(\rho)} G^{r}(\sigma, \rho) V_{r}(\rho)+\text { constant }
$$

where the constant is arbitrary. The Green's function $G^{r}$ is given by

$$
G^{r}(\sigma, \rho)=\sum_{A} \frac{1}{\omega^{A}} Y^{A}(\sigma) \partial^{r} Y_{A}(\rho)
$$

It is easy to check that this Green's function obeys

$$
\int d^{2} \sigma \sqrt{w(\sigma)} G^{r}(\sigma, \rho)=0, \quad D_{r}^{\prime} G^{r}(\sigma, \rho)=-\frac{1}{\sqrt{w(\sigma)}} \delta^{(2)}(\sigma, \rho)+1,
$$

(where $D_{r}^{\prime}$ denotes the covariant differentiation with respect to $\rho$ ) as one directly verifies from (2.30) and (2.19).

From (2.9) it is obvious that the Lie bracket corresponds to the structure constants of the (subgroup $G$ of the) area-preserving diffeomorphisms. We can now substitute the expansion (2.12) and thereby extract the structure constants of the group of area-preserving diffeomorphisms in this basis. From the completeness of the $Y_{A}$ (the function $Y_{0}$ does not play a role in what follows), we obtain

$$
\left\{Y_{A}, Y_{B}\right\}=f_{A B C} Y^{C} \text {, }
$$

so that the structure constants are defined by

$$
f_{A B C} \equiv \int d^{2} \sigma \sqrt{w(\sigma)} Y_{A}(\sigma)\left\{Y_{B}(\sigma), Y_{C}(\sigma)\right\}
$$

Note that $f_{A B C}$ is totally antisymmetric in $A, B$ and $C$.

As we have already indicated, the treatment of the Lorentz algebra in this formalism requires further tensors. One of them is the (fully symmetric) overlap integral between three $Y$ 's (readers curious to see where these definitions come from are invited to glance at the following section),

$$
d_{A B C} \equiv \int d^{2} \sigma \sqrt{w(\sigma)} Y_{A}(\sigma) Y_{B}(\sigma) Y_{C}(\sigma)
$$

A third tensor arises in the harmonic expansion of (2.29) for $V_{r} \propto Y \partial_{r} Y^{\prime}$. It is given by

$$
c_{A B C} \equiv-2 \int d^{2} \sigma \sqrt{w(\sigma)} \frac{w^{r s}(\sigma)}{\omega_{A}} \partial_{r} Y_{A}(\sigma) Y_{B}(\sigma) \partial_{s} Y_{C}(\sigma) .
$$

There are several important identities for these tensors that follow from the above definitions. For instance, the Jacobi identity is simply a consequence of the Jacobi identity (2.7) for the Lie brackets,

$$
f_{[A B}{ }^{E} f_{C] D E}=0
$$

where (2.32) has been used. Another relation follows immediately by partial integration,

$$
c_{A B C}+c_{A C B}=-2 d_{A B C} .
$$

Two identities similar to (2.36) are

$$
c_{D E}^{[A} f^{B C] E}=0
$$




$$
d_{A B C} f_{[D E}^{A} f^{B}{ }_{F] G}=0 .
$$

The proof of these identities is simple and follows from writing the left-hand sides as an integral of $\omega_{D}^{-1} \partial_{r} Y_{D}\left\{Y^{[B}, Y^{C}\right\} \partial^{r} Y^{A]}$ and $\left\{Y_{[D}, Y_{E}\right\}\left\{Y_{F]}, Y_{G}\right\} Y_{C}$, respectively. Subsequently one makes use of the Schouten identity, which in the present context expresses the vanishing of any tensor antisymmetric in three membrane indices.

The proof of the following identity requires a little more work,

$$
f_{A B}{ }^{E} c_{E C D}=c_{E A B} f_{C D}^{E}-2 f_{B D}{ }^{E} d_{A C E}+\sum_{\lambda} c_{\lambda A B} f_{C D}^{\lambda},
$$

where $f_{\lambda A B}$ and $c_{\lambda A B}$ are antisymmetric in $[A B]$ and defined by

$$
\begin{aligned}
& f_{\lambda A B}=\int d^{2} \sigma \sqrt{w(\sigma)} \Phi^{(\lambda) t}(\sigma) \partial_{t} Y_{B}(\sigma) Y_{A}(\sigma), \\
& c_{\lambda A B}=-2 \int d^{2} \sigma \varepsilon^{r s} \Phi_{r}^{(\lambda)}(\sigma) \partial_{s} Y_{B}(\sigma) Y_{A}(\sigma) .
\end{aligned}
$$

Observe that $f_{\lambda A B}$ is precisely the structure constant corresponding to the commutator (2.10). To derive (2.40) one substitutes the definitions (2.33) and (2.35) on the left-hand side, such that the contraction over the functions $Y_{E}$ takes the form $\partial_{r} Y^{E}(\sigma) \partial_{s} Y_{E}(\rho) \omega_{E}^{-1}$. This is just equal to $Y_{r}^{(1) E}(\sigma) Y_{s E}^{(1)}(\rho)$. Subsequently one uses the completeness relation (2.28), which leads directly to (2.40).

Before continuing let us examine the index structure of (2.40). Using the fact that the $c_{\lambda A B} f_{D C}{ }^{\lambda}$ is antisymmetric in $[A B]$ and $[C D]$, symmetrization of (2.40) in either one of these index pairs leads to

$$
f_{A(B}{ }^{E} d_{C D) E}=0
$$

where we have made use of (2.37). From this result we can rewrite (2.40) in the form

$$
f_{A B}{ }^{E} c_{E[C D]}-c_{E[A B]} f^{E}{ }_{C D}-2 f_{[D[B}{ }^{E} d_{A] C] E}=\sum_{\lambda} c_{\lambda A B} f^{\lambda}{ }_{C D} .
$$

As the left-hand side is now antisymmetric under the interchange of the index pairs $[A B]$ and $[C D]$, we conclude that also the right-hand side of (2.44) must exhibit this antisymmetry. This result can also be justified on the basis of independent geometric arguments.

Furthermore, one may contract (2.40) by $f_{F G}{ }^{c}$ and antisymmetrize in the three indices $[F G D]$. Using the Jacobi identity (2.36), this yields

$$
f_{A B}{ }^{E}\left(c_{E C}{ }^{[D} f^{F G] C}\right)=-2 d_{A C E} f^{E B[D} f^{F G] C} .
$$

This result is obviously consistent with (2.38) and (2.39). Observe that in the derivation of (2.45) we have also used the Jacobi identity for structure constants that involve one index $\lambda$.

$$
f_{[A B}^{E} f_{C] E \lambda}=0 .
$$

Clearly there are more identities for products of the various tensors, such as

$$
d_{E A[B} d_{C] D}{ }^{E}=-\eta_{A[B} \eta_{C] D} .
$$

However, these identities are not needed in what follows, and we will not further elaborate on them.

Let us now return to (2.36) and (2.43), which suggest that $f$ and $d$ are invariant 
tensors under the subgroup $G$ of the area-preserving diffeomorphisms. Actually, it is easy to see that $f$ and $d$, as well as $\eta_{A B}$, defined in (2.15), are invariant under all area-preserving transformations, not just under those contained in $G$. This follows from the observation that $f, d$ and $\eta$ are defined in terms of integrals that are invariant under general reparametrizations, provided that one also transforms the metric $w_{r s}$ in the usual manner. On the other hand, $w_{r s}$ is supposed to be a given metric. However, since $f$ does not depend on $w_{r s}$ while $d$ and $\eta$ depend only on the determinant of $w_{r s}$ which is invariant under area-preserving diffeomorphisms, $f, d$ and $\eta$ must be invariant under the change of the functions $Y_{A}$ induced by these diffeomorphisms.

However, the above arguments are not applicable to the tensor $c_{A B C}$, because it depends explicitly on the metric; consequently we have

$$
\delta c_{A B C}=2 \int d^{2} \sigma \sqrt{w(\sigma)} \frac{1}{\omega_{A}} \partial_{r} Y_{A}(\sigma) Y_{B}(\sigma) \partial_{s} Y_{C}(\sigma)\left(D^{r} \xi^{s}+D^{s} \xi^{r}\right)
$$

which does not vanish. On the other hand, the covariant variation suggested by the index structure of $c_{A B C}$, gives

$$
\begin{aligned}
\delta c_{A B C} & \equiv \xi^{E}\left(f_{A E}^{D} c_{D B C}+f_{B E}^{D} c_{A D C}+f_{C E}^{D} c_{A B D}\right) \\
& =\xi^{E}\left(f_{B C}{ }^{D}\left(c_{D A E}+c_{A D E}\right)+f_{B C}{ }^{\lambda} c_{\lambda A E}\right),
\end{aligned}
$$

where we have made use of (2.38) and (2.44). For the transformations generated by the harmonic vector fields, we can derive a similar result, which takes the same form as (2.49) upon changing the index $E$ into $\lambda$. This leads to the definition of new tensors $c_{A B \lambda}$ and $c_{\lambda A \lambda^{\prime}}$. Furthermore we note that there are identities similar to (2.38)-(2.40) in which one index is replaced by $\lambda$ (for example, see (2.46)).

In Sect. 3 we will argue that $c_{A B C}$ is only relevant up to terms proportional to $f_{B C}{ }^{D}$. On the other hand, (2.49) shows that $c_{A B C}$ is invariant up to precisely such a term. This would seem to suggest that $c_{A B C}$ can be made invariant by making a suitable modification proportional to $f_{B C}{ }^{D}$. Under such a modification the identities (2.37) and (2.38) would remain valid by virtue of the Jacobi identity (2.36), whereas (2.40) and (2.44) would acquire an extra term proportional to $f_{A B}{ }^{E} d_{C D} F$. Assuming the existence of the Killing-Cartan metric

$$
g_{A B}=-f_{A C}^{D} f_{D B}^{C},
$$

one can indeed write down a modified tensor $C_{A B C}^{\prime}$, which is formally invariant. It reads

$$
c_{A B C}^{\prime}=c_{A B C}-f_{B C}^{D} c_{D E F} f^{E F G}\left(g^{-1}\right)_{G A} .
$$

From (2.49) and (2.50) one can easily verify the invariance of $c_{A B C}^{\prime}$. However, in the case at hand, these conditions cannot be applied because the Killing-Cartan metric is singular for the group $G$ of area-preserving diffeomorphisms. Therefore no modification will render the tensor $c_{A B C}$ invariant. ${ }^{2}$ On the other hand, the

\footnotetext{
2 This conclusion is in agreement with a more general result in Lie algebra cohomology, according to which there are only nontrivial cohomology classes in representations where the second-order Casimir operator is invertible. See, e.g., [17]
} 
modification is possible if the group $G$ is truncated to a finite group such as $S U(N)$. Then any tensor with the same properties as $c_{A B C}$ is actually expressible as a linear combination of the invariant three-index tensors of the finite group, up to the modification given in (2.51). Therefore it appears that the existence of a tensor $c_{A B C}$ different from either $f_{A B C}$ or $d_{A B C}$ is a genuine property of the infinite-dimensional group $G$.

This brings us to the question of how the definitions and results described so far are affected when the group $G$ is replaced by a finite group. As already mentioned, such an approximation is possible for spherical and toroidal membranes, where the structure constants $f_{A B C}$ can be obtained from the ordinary $S U(N)$ structure constants in the limit $N \rightarrow \infty$. We have demonstrated the necessity of further numerical tensors for the Lorentz generators, and this raises the question whether these, too, can be approximated by $S U(N)$ tensors. The tensor $d_{A B C}$ defined in (2.34) evidently corresponds to the symmetric three-index tensor of $S U(N)$, and in the appendices we will confirm this expected correspondence (which, incidentally shows that the $S U(N)$ groups are the only finite-dimensional simple Lie groups that can possibly serve as a regulator group, since no other simple Lie groups admit an invariant symmetric three-index tensor). As for the tensor $c_{A B C}$, the arguments presented above show that it has no counterpart for finite-dimensional groups. In accordance with these arguments we can try to represent it as a linear combination of the known $S U(N)$ invariants $d_{A B C}$ and $f_{A B C}$. Quite independently of the explicit representation, however, it is impossible for finite $N$ to simultaneously satisfy all of the identities that we have derived in this section; it is even impossible to satisfy the ones involving only $d_{A B C}$ and $f_{A B C}$. This is most conveniently checked by means of the explicit formulae of Appendix A. For instance, (2.39) and (2.47) are invalid for finite $N$; substitution of the relevant expressions in Appendix $A$ shows the violations to be of order $O\left(1 / N^{2}\right)$ for finite $N$. As the identities involve only invariant tensors, this conclusion is independent of the explicit basis chosen for the $S U(N)$ matrices. To be sure, there is a certain ambiguity in the choice of $c_{A B C}$ for finite $N$. Since it has no proper $S U(N)$ analog and since the identities we relied on in deriving (2.51) are anyhow violated for finite $N$, we can just as well regulate it by any tensor whose components differ from their exact values by $O\left(1 / N^{2}\right.$ ) (further details are given in the appendices). In this way, it is possible to satisfy all identities up to terms of order $O\left(1 / N^{2}\right)$.

\section{Lorentz Invariance}

In this section we will apply the formalism developed in the foregoing section to establish the Lorentz invariance of the classical supermembrane formulated in the light-cone gauge and to show that it holds independently of the topology of the membrane. We will not repeat the basic results about supermembranes here but rather assume that the reader is familiar with them (see e.g. [1]); in particular, we refer the reader to [7] for our conventions and notation as well as a derivation of the results needed here.

The canonical Hamiltonian $H$ in the light-cone gauge is given by 


$$
H=-\int d^{2} \sigma P^{-}(\sigma)=\frac{1}{P_{0}^{+}} \int d^{2} \sigma \sqrt{w(\sigma)}\left(\frac{1}{2} w^{-1} \vec{P}^{2}+\frac{1}{4}\left(\left\{X^{a}, X^{b}\right\}\right)^{2}-\frac{i}{2} \theta \gamma_{a}\left\{X^{a}, \theta\right\}\right) .
$$

Here, $\vec{P}(\sigma)$ denotes the canonical momenta conjugate to the (transverse) coordinates $\vec{X}(\sigma)$, which transform as a density under reparametrizations of $\sigma^{r} ; \theta(\sigma)$ are the fermionic coordinates, which transform as a spinor under the transverse rotations and as a scalar under reparametrizations. ${ }^{3}$ Our choice for these coordinates is such that the only nonvanishing Dirac brackets take the form

$$
\begin{aligned}
\left(X^{a}(\sigma), P^{b}(\rho)\right)_{D B} & =\delta^{a b} \delta^{(2)}(\sigma, \rho), \\
\left(\theta_{\alpha}(\sigma), \theta_{\beta}(\rho)\right)_{D B} & =-\frac{i}{\sqrt{w(\sigma)}} \delta_{\alpha \beta} \delta^{(2)}(\sigma, \rho) .
\end{aligned}
$$

The quantity $P_{0}^{+}$represents the total momentum of the supermembrane in one of the directions along the light-cone; as indicated in (3.1) the other momentum component along the light-cone is related to the Hamiltonian. Regarding the light-cone components of the coordinate vector, $X^{+}$is related to the time $\tau$, the dependence on which we have suppressed above, while $X^{-}$does not explicitly appear in the Hamiltonian, but is determined through the condition

$$
\partial_{r} X^{-}(\sigma)=-\frac{1}{P_{0}^{+}}\left(\frac{1}{\sqrt{w(\sigma)}} \vec{P}(\sigma) \cdot \partial_{r} \vec{X}(\sigma)+\frac{i}{2} \theta(\sigma) \partial_{r} \theta(\sigma)\right) .
$$

As was first observed by Goldstone [2] this condition can be solved by means of the Green's function $G^{r}$ introduced in Sect. 2, provided the following conditions hold (cf. (2.22)),

$$
\begin{array}{r}
\left\{\frac{\vec{P}(\sigma)}{\sqrt{w(\sigma)}}, \vec{X}(\sigma)\right\}+\frac{i}{2}\{\theta(\sigma), \theta(\sigma)\}=0 \\
\oint\left(\vec{P}(\sigma) \cdot \partial_{r} \vec{X}(\sigma)+\frac{i}{2} \sqrt{w(\sigma)} \theta(\sigma) \partial_{r} \theta(\sigma)\right) d \sigma^{r}=0 .
\end{array}
$$

We then obtain (cf. (2.29))

$$
X^{-}(\sigma)=q^{-}-\frac{1}{P_{0}^{+}} \int d^{2} \rho G^{r}(\sigma, \rho)\left(\vec{P}(\rho) \cdot \partial_{r} \vec{X}(\rho)+\frac{i}{2} \sqrt{w(\rho)} \theta(\rho) \partial_{r} \theta(\rho)\right),
$$

where $q^{-}$denotes the center-off-mass coordinate of the membrane, which is canonically conjugate to $P_{0}^{+}$. Therefore we have an additional nonvanishing Dirac bracket,

$$
\left(q^{-}, P_{0}^{+}\right)_{D B}=1
$$

\footnotetext{
${ }^{3}$ If the membrane moves in a 11-dimensional space-time, the fermionic coordinates $\theta$ are thus 16-component spinors of $S O(9)$. In contrast with the coordinates used in [7], we have rescaled $\theta$ by $\sqrt{P_{0}^{+}}$times a numerical factor. The gamma matrices $\gamma_{a}$ are real and symmetric
} 
The part of phase space that satisfies the constraints (3.4) and (3.5) will be referred to as the "physical subspace."

The supermembrane is invariant under Lorentz transformations and spacetime supersymmetry, although not all these invariances are manifest in the light-cone gauge. Furthermore, the invariance under general diffeomorphisms of the three-dimensional world tube is broken in this gauge and one is left with a residual invariance under area-preserving diffeomorphisms, which we have extensively discussed in Sect. 2. According to [18] the first-class constraints (3.4) and (3.5) are also the generators of the area-preserving diffeomorphisms. These generators therefore take the form

$$
\begin{aligned}
\varphi(\sigma) & =-\left\{\frac{\vec{P}(\sigma)}{\sqrt{w(\sigma)}}, \vec{X}(\sigma)\right\}-\frac{i}{2}\{\theta(\sigma), \theta(\sigma)\}, \\
\varphi^{\lambda} & =-\int d^{2} \sigma \Phi^{(\lambda) r}(\sigma)\left(\vec{P}(\sigma) \cdot \partial_{r} \vec{X}(\sigma)+\frac{i}{2} \sqrt{w(\sigma)} \theta(\sigma) \partial_{r} \theta(\sigma)\right) .
\end{aligned}
$$

To calculate the Lorentz and supersymmetry generators of the supermembrane in the light-cone gauge, we apply the Noether procedure to the original supermembrane action, pass to light-cone coordinates and impose the light-cone gauge conditions. This straightforward but somewhat tedious calculation leads to the following result for the Lorentz generators:

$$
\begin{aligned}
M^{a b} & =\int d^{2} \sigma\left(-P^{a} X^{b}+P^{b} X^{a}-\frac{i}{4} \theta \gamma^{a b} \theta\right) \\
M^{+-} & =\int d^{2} \sigma\left(-P^{+} X^{-}+P^{-} X^{+}\right) \\
M^{+a} & =\int d^{2} \sigma\left(-P^{+} X^{a}+P^{a} X^{+}\right), \\
M^{-a} & =\int d^{2} \sigma\left(P^{a} X^{-}-P^{-} X^{a}-\frac{i}{4 P_{0}^{+}} \theta \gamma^{a b} \theta P_{b}-\frac{i \sqrt{w}}{8 P_{0}^{+}}\left\{X_{b}, X_{c}\right\} \theta \gamma^{a b c} \theta\right) .
\end{aligned}
$$

The supersymmetry generators read

$$
\begin{aligned}
& Q^{+}=\frac{1}{\sqrt{P_{0}^{+}}} \int d^{2} \sigma\left(P^{a} \gamma_{a}+\frac{\sqrt{w}}{2}\left\{X^{a}, X^{b}\right\} \gamma_{a b}\right) \theta \\
& Q^{-}=\sqrt{P_{0}^{+}} \int d^{2} \sigma \sqrt{w(\sigma)} \theta
\end{aligned}
$$

where $Q^{+}$and $Q^{-}$transform according to inequivalent spinor representations of the transverse rotation group.

Following the analysis of the previous section, we expand the membrane coordinates into a complete set of orthonormal functions, so that all relevant quantities can be expressed with the help of the tensors $f_{A B C}, d_{A B C}, c_{A B C}$ and the metric $\eta_{A B}$. For instance, the generators of the area-preserving reparametrizations now become 


$$
\begin{gathered}
\varphi_{A}=f_{A B C}\left(\vec{X}^{B} \cdot \vec{P}^{C}-\frac{i}{2} \theta^{B} \theta^{C}\right), \\
\varphi_{\lambda}=f_{\lambda B C}\left(\vec{X}^{B} \cdot \vec{P}^{C}-\frac{i}{2} \theta^{B} \theta^{C}\right),
\end{gathered}
$$

and the constraints

$$
\varphi_{A}=0, \quad \varphi^{\lambda}=0
$$

hold on the physical subspace.

In this formulation the canonical brackets are given by

$$
\begin{array}{ll}
\left(X_{A}^{a}, P_{B}^{b}\right)_{D B}=\delta^{a b} \eta_{A B}, & \left(\theta_{\alpha A}, \theta_{\beta B}\right)_{D B}=-i \delta_{\alpha \beta} \eta_{A B}, \\
\left(q^{-}, P_{0}^{+}\right)_{D B}=1, & \left(\theta_{\alpha 0}, \theta_{\beta 0}\right)_{D B}=-i \delta_{\alpha \beta} .
\end{array}
$$

The solution (3.3) for the light-cone coordinate $X^{-}$can now be written explicitly with the help of the coefficient $c_{A B C}$ defined in Sect. 2,

$$
X_{A}^{-}=-\frac{1}{P_{0}^{+}}\left(\vec{P}_{0} \cdot \vec{X}_{A}+\frac{i}{2} \theta_{0} \theta_{A}\right)+\frac{1}{2 P_{0}^{+}} c_{A B C}\left(\vec{P}^{B} \cdot \vec{X}^{C}+\frac{i}{2} \theta^{B} \theta^{C}\right) .
$$

In view of the remarks made towards the end of Sect. 2, it is clear that $X_{A}^{-}$does not transform covariantly under the group of area-preserving diffeomorphisms. Using (2.49) we find

$$
\delta X_{A}^{-}=\xi^{B} f_{A B}^{C} X_{C}^{-}+\frac{\xi^{B}}{2 P_{0}^{+}}\left(\left(c_{C A B}+c_{A C B}\right) \varphi^{C}+c_{\lambda A B} \varphi^{\lambda}\right),
$$

and a similar result for the diffeomorphisms generated by harmonic vectors. However, the anomalous terms in the transformation rules of $X_{A}^{-}$are proportional to the constraints $\varphi^{A}$ and $\varphi^{\lambda}$, so that $X_{A}^{-}$transforms covariantly in the physical subspace. Of course, the same remark applies to the Lorentz generators $M^{-a}$, which depend on $X_{A}^{-}$.

The Hamiltonian (3.1) reads in this basis,

$$
H=\frac{\vec{P}_{0}^{2}}{2 P_{0}^{+}}+\frac{\mathscr{M}^{2}}{2 P_{0}^{+}},
$$

where $\mathscr{M}$ is the invariant mass of the supermembrane, which is given by

$$
\mathscr{M}^{2}=\vec{P}_{A}^{2}+\frac{1}{2}\left(f_{A B C} X_{a}^{B} X_{b}^{C}\right)^{2}-i f_{A B C} \theta^{A} \gamma^{a} X_{a}^{B} \theta^{C} .
$$

Observe that $\mathscr{M}$ does not depend on the center-of-mass coordinates and momenta, and neither does it depend on $P_{0}^{+}$.

It is straightforward to substitute the mode expansions and (3.16) into the Lorentz generators listed in (3.10). The first three generators take a simple form,

$$
\begin{aligned}
M^{a b} & =-P_{0}^{a} X_{0}^{b}+P_{0}^{b} X_{0}^{a}-\frac{i}{4} \theta_{0} \gamma^{a b} \theta_{0}-P_{A}^{a} X^{b A}+P_{A}^{b} X^{a A}-\frac{i}{4} \theta_{A} \gamma^{a b} \theta^{A}, \\
M^{+-} & =-P_{0}^{+} q^{-}-H \tau, \\
M^{+a} & =-P_{0}^{+} X_{0}^{a}+\tau P_{0}^{a},
\end{aligned}
$$


where we have substituted $X^{+}=\tau$. It is convenient to write $M^{-a}$, which contains many more terms, in the following form:

$$
M^{-a}=\left(M^{-a}\right)^{(0)}+\frac{1}{P_{0}^{+}}\left(P_{0 b} \tilde{M}^{a b}-\frac{i}{2} \theta_{0} \gamma^{a} \tilde{Q}^{+}\right)+\frac{1}{P_{0}^{+}} \tilde{M}^{-a},
$$

where $\left(M^{-a}\right)^{(0)}$ represents the contributions of the center-of-mass modes,

$$
\left(M^{-a}\right)^{(0)}=q^{-} P_{0}^{a}+X_{0}^{a} H-\frac{i}{8 P_{0}^{+}} \theta_{0} \gamma^{a b} \theta_{0} P_{0}^{b}
$$

and $\tilde{Q}^{+}, \tilde{M}^{a b}$ and $\tilde{M}^{-a}$ refer to contributions of the nonzero modes; those are defined by

$$
\begin{aligned}
& \widetilde{Q}^{+}=\left(P_{A}^{a} \gamma_{a}+\frac{1}{2} f_{A B C} X_{a}^{B} X_{b}^{C} \gamma^{a b}\right) \theta^{A}, \\
& \tilde{M}^{a b}=-P_{A}^{a} X^{b A}+P_{A}^{b} X^{a A}-\frac{i}{4} \theta_{A} \gamma^{a b} \theta^{A}, \\
& \tilde{M}^{-a}=\frac{1}{2} d^{A B C} X_{A}^{a}\left(\vec{P}_{B} \cdot \vec{P}_{C}+\frac{1}{2}\left(f_{B}{ }^{D E} X_{D}^{b} X_{E}^{c}\right)\left(f_{C}{ }^{F G} X_{F}^{b} X_{G}^{c}\right)-i f_{C}{ }^{D E} X_{D}^{b} \theta_{B} \gamma_{b} \theta_{E}\right) \\
& -\frac{1}{4} i d^{A B C} P_{A b} \theta_{B} \gamma^{a b} \theta_{C}+\frac{1}{2} c^{A B C} P_{A}^{a}\left(\vec{P}_{B} \cdot \vec{X}_{C}+\frac{1}{2} i \theta_{B} \theta_{C}\right) \\
& -\frac{1}{8} i f^{A B C} d_{A}^{D E} X_{B b} X_{C c} \theta_{D} \gamma^{a b c} \theta_{E} .
\end{aligned}
$$

In principle, the construction of the Lorentz generators ensures that they are conserved charges i.e.,

$$
\frac{d}{d \tau} M=\frac{\partial}{\partial \tau} M+(M, H)_{D B}=0
$$

where $M$ generically denotes all the Lorentz generators. ${ }^{4}$ Explicit verification of (3.28) can thus be viewed as a check on the consistency of the light-cone gauge. However, in the formulation that we employ the classical check of the Lorentz invariance is reduced to a purely algebraic problem for the tensors defined in the previous section: what we intend to show is that Lorentz invariance is a consequence of some of the tensor identities derived in Sect. 2. This has the advantage that we can explicitly see where the Lorentz invariance breaks down in the $S U(N)$ approximation.

The verification of (3.28) for the generators $M^{a b}, M^{+-}$and $M^{+a}$ is straightforward, and does not depend on the properties of the various tensors. The proof of the $\tau$-independence of $M^{-a}$ is more involved. In order to simplify the discussion somewhat, we first note that the Dirac bracket of $\left(M^{-a}\right)^{(0)}$ and $H$ vanishes. Using the decomposition (3.23) one can then easily show that $M^{-a}$ is conserved, provided that

$$
\left(\tilde{M}^{a b}, \mathscr{M}^{2}\right)_{D B}=0
$$

\footnotetext{
${ }^{4}$ In principle one should use the total Hamiltonian, but as the Lorentz generators are invariant under area-preserving diffeomorphisms in the physical subspace (cf. (3.17)) it is sufficient to consider the canonical Hamiltonian
} 


$$
\begin{aligned}
\left(\tilde{Q}^{+}, \mathscr{M}^{2}\right)_{D B} & =0 \\
\left(\tilde{M}^{-a}, \mathscr{M}^{2}\right)_{D B} & =0 .
\end{aligned}
$$

The first equation is obviously satisfied as a consequence of the manifest invariance of $\mathscr{M}^{2}$ under transverse rotations. While the second equation represents the well-known fact that the invariant supermembrane mass is supersymmetric (see, e.g. [7]), its explicit verification is not entirely straightforward, and depends on the Jacobi identity (2.36) and an identity for anticommuting spinors,

$$
\begin{aligned}
& \theta_{1} \gamma^{b} \theta_{2} \theta_{3} \gamma_{a b} \theta_{4}+\theta_{3} \gamma^{b} \theta_{4} \theta_{1} \gamma_{a b} \theta_{2}-\theta_{1} \gamma^{b} \theta_{4} \theta_{3} \gamma_{a b} \theta_{2} \\
& \quad-\theta_{3} \gamma^{b} \theta_{2} \theta_{1} \gamma_{a b} \theta_{4}+2 \theta_{4} \theta_{2} \theta_{3} \gamma_{a} \theta_{1}-2 \theta_{1} \theta_{3} \theta_{2} \gamma_{a} \theta_{4}=0 .
\end{aligned}
$$

This identity is derived from a similar identity for Lorentz spinors in the covariant formulation of the supermembrane [5]. As is well-known, the necessity for these identities leads to the restriction that supermembranes can only live in a $d=4,5,7$ or 11 dimensional space-time. Using (3.32) one derives

$$
\left(\tilde{Q}^{+}, \mathscr{M}^{2}\right)_{D B}=2 \theta_{A} \varphi^{A},
$$

so that (3.30) is satisfied in the physical subspace.

Finally, we are left with the proof of (3.31). We find it convenient to write the result in the following way,

with

$$
\left(\tilde{M}^{-a}, \mathscr{M}^{2}\right)_{D B}=A_{1}+A_{2}+A_{3}+A_{4}+A_{5}+A_{6}+A_{7},
$$

$$
\begin{aligned}
A_{1}= & P_{A}^{a}\left(c^{A B C}+d^{A B C}\right) \vec{P}_{B} \cdot \vec{P}_{C}, \\
A_{2}= & P_{A}^{a}\left(\frac{1}{2} d^{A B C} f_{B}{ }^{D E} f_{C}{ }^{F G} \vec{X}_{D} \cdot \vec{X}_{F} \vec{X}_{E} \cdot \vec{X}_{G}\right. \\
& \left.-c^{A B C} f^{D E F} f_{B D}{ }^{G} \vec{X}_{C} \cdot \vec{X}_{F} \vec{X}_{E} \cdot \vec{X}_{G}\right), \\
A_{3}= & i P_{A}^{a}\left(-d^{A B C} f_{C}{ }^{D E} X_{D}^{b} \theta_{B} \gamma_{b} \theta_{E}-\frac{1}{2} c^{A B C} f_{B}{ }^{D E} X_{C}^{b} \theta_{D} \gamma_{b} \theta_{E}\right. \\
& \left.-\frac{1}{2} c^{A B C} f_{C}{ }^{D E} X_{D}^{b} \theta_{B} \gamma_{b} \theta_{E}+\frac{1}{2} c^{A B C} f_{B}{ }^{D E} X_{D}^{b} \theta_{C} \gamma_{b} \theta_{E}\right), \\
A_{4}= & \frac{1}{2} i\left(d^{B D E} f^{A G F} f_{A B}{ }^{C} X_{G b} \vec{X}_{C} \cdot \vec{X}_{F} \theta_{D} \gamma^{a b} \theta_{E}\right. \\
& -c^{B D E} f^{A F G} f_{A B}{ }^{C} X_{F}^{a} \vec{X}_{C} \cdot \vec{X}_{G} \theta_{D} \theta_{E} \\
& +d^{A B C} f_{A}{ }^{F G} f_{C}{ }^{D E} X_{F b} X_{G c} X_{D d} \theta_{B} \gamma^{a b c} \gamma^{d} \theta_{E} \\
& \left.+2 d^{A B C} f_{C}{ }^{D E} f_{E}{ }^{F G} X_{A}^{a} X_{D}^{b} X_{F}^{c} \theta_{B} \gamma_{b} \gamma_{c} \theta_{G}\right), \\
A_{5}= & X_{A}^{a}\left(2 d^{A B C} f_{B}{ }^{D E} f_{C}{ }^{F G} \vec{X}_{D} \cdot \vec{X}_{F} \vec{X}_{E} \cdot \vec{P}_{G}\right. \\
& -2 d^{A B C} f_{E}^{G F} f_{C}{ }^{E D} \vec{X}_{F} \cdot \vec{P}_{B} \vec{X}_{D} \cdot \vec{X}_{G} \\
& \left.-c^{B C D} f^{A E F} f_{B E}{ }^{G} \vec{X}_{D} \cdot \vec{P}_{C} \vec{X}_{F} \cdot \vec{X}_{G}\right), \\
A_{6}= & -i X_{A}^{a} d^{A B C}\left(f_{C}{ }^{D E} P_{D}^{b} \theta_{B} \gamma_{b} \theta_{E}+f_{C}{ }^{D E} P_{B}^{b} \theta_{D} \gamma^{b} \theta_{E}\right) \\
& +i d^{A B C}\left(f_{B}{ }^{D E} P_{C b} X_{D c} \theta_{A} \gamma^{a b} \gamma^{c} \theta_{E}-\frac{1}{2} f_{A}{ }^{D E} P_{D b} X_{E c} \theta_{B} \gamma^{a b c} \theta_{C}\right) \\
& -\frac{1}{2} i c^{A B C} f_{A}{ }^{D E} \vec{P}_{B} \cdot \vec{X}_{C} \theta_{D} \gamma^{a} \theta_{E}, \\
A_{7}= & \frac{1}{4}\left(c^{A B E} f_{A}^{C D}+2 d^{A B C} f_{A}{ }^{D E}\right) \theta_{B} \theta_{E} \theta_{C} \gamma^{a} \theta_{D} .
\end{aligned}
$$

Observe that the evaluation of the $\theta^{4}$-terms requires the use of the identity (3.32).

We will now show that (3.34) vanishes in the physical subspace by virtue of the 
tensor identities derived in Sect. 2. Using (2.37) and (2.38) one first shows that $A_{1}, A_{2}$ and $A_{3}$ vanish. Subsequently, one rewrites $A_{4}$ and $A_{6}$ in the following form:

$$
\begin{aligned}
& A_{4}=i f_{A}{ }^{F C}\left(d^{A B D} f_{C}{ }^{G E}-\frac{1}{2} c^{A B E} f_{C}{ }^{D G}\right) X_{D}^{a} \vec{X}_{F} \cdot \vec{X}_{G} \theta_{B} \theta_{E}, \\
& A_{6}=-i\left(\frac{1}{2} c_{A}{ }^{B E} f^{A C D}+d_{A}{ }^{B C} f^{A D E}\right) \theta_{C} \gamma^{a} \theta_{D} \vec{X}_{E} \cdot \vec{P}_{B},
\end{aligned}
$$

where, for $A_{4}$, we made use of (2.36-39) and (2.43), while, for $A_{6}$, we only used (2.43). Combining the previous results, and using again (2.36) and (2.43), one finds

$$
\begin{aligned}
\left(\tilde{M}^{-a}, \mathscr{M}^{2}\right)_{D B}= & \left\{-d_{C}{ }^{D G} f^{A C F} f_{A}{ }^{E B} X_{F}^{a} \vec{X}_{D} \cdot \vec{X}_{G}\right. \\
& \left.+\left(f_{C}{ }^{F G} X_{F}^{a} \vec{X}_{D} \cdot \vec{X}_{G}-\frac{i}{2} \theta_{C} \gamma^{a} \theta_{D}\right)\left(c^{A B E} f_{A}{ }^{C D}-2 d^{A B D} f_{A}{ }^{C E}\right)\right\} \\
& \times\left(\vec{X}_{E} \cdot \vec{P}_{B}-\frac{i}{2} \theta_{E} \theta_{B}\right) .
\end{aligned}
$$

It is now easy to see that (3.38) becomes proportional to the constraints $\varphi^{A}$ and $\varphi^{\lambda}$, as result of $(2.40)$,

$$
\left(\tilde{M}^{-a}, \mathscr{M}^{2}\right)_{D B}=-\left(f_{B}{ }^{D E} X_{D}^{a} \vec{X}_{C} \cdot \vec{X}_{E}-\frac{i}{2} \theta_{B} \gamma^{a} \theta_{C}\right)\left(c^{A B C} \varphi_{A}+c^{\lambda B C} \varphi_{\lambda}\right),
$$

so that also the Lorentz boost operators $M^{-a}$ are conserved in the physical subspace. We should stress that this result is valid irrespective of the topology of the membrane. In the $S U(N)$ approximation we get of course additional terms and Lorentz invariance is inevitably broken for finite $N$. As the relations $(2.37-40)$ hold for finite $N$ modulo terms of order $O\left(1 / N^{2}\right)$, these additional terms are of order $O\left(1 / N^{2}\right)$, too. This shows that in the limit $N \rightarrow \infty$ the Lorentz symmetry is restored.

From the fact that the Lorentz generators are conserved, it follows that these generators must close with respect to the Dirac brackets, at least in the physical subspace. On the other hand, an explicit verification of this closure seems to require various new identities, such as identities for products of the $c$-tensors. As the closure is already guranteed by the calculation of this section, we must conclude that these new identities are already contained in the set of identities used above. Another issue concerns the evaluation of the quantum Lorentz algebra, but as we have pointed out in Sect. 1, this requires more insight in the structure of the quantum divergences of supermembranes.

\section{Appendix A: Area-Preserving Diffeomorphisms on $\boldsymbol{T}^{2}$}

Choosing torus coordinates $\sigma=\left(\sigma_{1}, \sigma_{2}\right)$ with $\sigma_{1}, \sigma_{2} \in[0,2 \pi)$, we label the basis functions $Y_{A}$ by two-dimensional nonzero vectors with integer components $\mathbf{A}=\left(A_{1}, A_{2}\right)$. They are given by

$$
Y_{\mathbf{A}}(\sigma)=\exp \left(i\left(A_{1} \sigma_{1}+A_{2} \sigma_{2}\right)\right)=\left(Y^{\mathbf{A}}(\sigma)\right)^{*} .
$$


With $w_{r s}(\sigma)=\left(4 \pi^{2}\right)^{-1} \delta_{r s}$ it is straightforward to check that the basis functions are normalized and to compute the various tensors defined in Sect. 2. We find

$$
\begin{aligned}
\eta_{\mathbf{A B}} & =\delta_{\mathbf{A}+\mathbf{B}}, \\
f_{\mathbf{A B C}} & =-4 \pi^{2}(\mathbf{A} \times \mathbf{B}) \delta_{\mathbf{A}+\mathbf{B}+\mathbf{C}}, \\
d_{\mathbf{A B C}} & =\delta_{\mathbf{A}+\mathbf{B}+\mathbf{C}} \\
c_{\mathbf{A B C}} & =2 \frac{\mathbf{A} \cdot \mathbf{C}}{\mathbf{A} \cdot \mathbf{A}} \delta_{\mathbf{A}+\mathbf{B}+\mathbf{C}},
\end{aligned}
$$

where $\mathbf{A} \times \mathbf{B} \equiv A_{1} B_{2}-A_{2} B_{1}$. The normalized harmonic vectors are

$$
\Phi_{r}^{(\lambda)}(\sigma)=\frac{1}{2 \pi} \delta_{r}^{\lambda} . \quad(\lambda=1,2) .
$$

From these we compute the tensors (2.41) and (2.42),

$$
\begin{aligned}
& f_{\lambda \mathbf{B C}}=2 \pi i C_{\lambda} \delta_{\mathbf{B}+\mathbf{C}}, \\
& c_{\lambda \mathbf{B C}}=-4 \pi i \varepsilon^{\lambda r} C_{r} \delta_{\mathbf{B}+\mathbf{C}} .
\end{aligned}
$$

The identities of Sect. 2 and, in particular, the noninvariance of $c$ can now be explicitly verified.

It has been recognized only recently that the structure constants of the torus algebra can also be approximated by $S U(N)$ structure constants $[19,10,12]$. The essential trick is to exploit 't Hooft's twist matrices [20]. These are $S U(N)$ matrices $\Omega_{1}, \Omega_{2}$, obeying [20,21]

$$
\Omega_{1} \Omega_{2}={ }_{z} \Omega_{2} \Omega_{1},
$$

where the phase factor $z$ must satisfy $z^{N}=1$ (explicit representations for these matrices can be found in $[20,21])$. For later convenience we choose $N$ odd and put

$$
z \equiv \exp \left(-\frac{4 \pi i}{N}\right) .
$$

To each vector $\mathbf{A}$ we assign a $N \times N$ matrix

$$
T_{\mathrm{A}} \equiv N z^{(1 / 2) A_{1} A_{2}} \Omega_{2}^{A_{1}} \Omega_{1}^{A_{2}} .
$$

Making use of the explicit representation of the twist matrices given in $[20,21]$ we can now show that

$$
\begin{aligned}
& T_{\mathbf{A}}^{\dagger}=T_{-\mathbf{A}}, \quad \operatorname{Tr} T_{\mathbf{A}}=0, \\
& T_{\mathbf{A}}=T_{\mathbf{B}} \quad \text { iff } \quad A_{r}=B_{r} \bmod N .
\end{aligned}
$$

According to (A.13) we can restrict the vectors $\mathbf{A}$ to some fundamental lattice, for instance the sub-lattice defined by $A_{1}, A_{2}=0, \ldots, N-1$, with the exception of the origin $A_{1}=A_{2}=0$. Hence we conclude that there are precisely $N^{2}-1$ traceless independent matrices $T_{\mathrm{A}}$, which we will use as the generators of $S U(N)$. Furthermore, if we also include the identity matrix, $T_{0}=N 1$, we have a complete 
set of $N \times N$ matrices, which close under multiplication. One easily derives

$$
\begin{aligned}
T_{\mathbf{A}} T_{\mathbf{B}} & =N z^{-(1 / 2)(\mathbf{A} \times \mathbf{B})} T_{\mathbf{A}+\mathbf{B}}, \\
\operatorname{Tr}\left(T_{\mathbf{A}} T_{\mathbf{B}}\right) & =N^{3} \delta_{\mathbf{A}+\mathbf{B}},
\end{aligned}
$$

where $\delta_{\mathbf{A}+\mathbf{B}}$ equals 1 iff $A_{\mathbf{r}}=B_{\mathbf{r}} \bmod N$, and zero otherwise. From (A.15) we define an $S U(N)$-invariant tensor $\tilde{\eta}_{\mathrm{AB}}$,

$$
\tilde{\eta}_{\mathbf{A B}} \equiv \frac{1}{N^{3}} \operatorname{Tr}\left(T_{\mathbf{A}} T_{\mathbf{B}}\right)=\delta_{\mathbf{A}+\mathbf{B}}
$$

which coincides with (A.2). The coefficients appearing in the product rule (A.14) decompose into two $S U(N)$-invariant tensors. One corresponds to the $S U(N)$ structure constants

$$
\tilde{f}_{\mathbf{A B C}} \equiv-\frac{i}{N^{3}} \operatorname{Tr}\left(\left[T_{\mathbf{A}}, T_{\mathbf{B}}\right] T_{\mathbf{C}}\right)=2 N \sin \frac{2 \pi(\mathbf{A} \times \mathbf{B})}{N} \delta_{\mathbf{A}+\mathbf{B}+\mathbf{C}}
$$

the other to the symmetric invariant tensor

$$
\tilde{d}_{\mathbf{A B C}} \equiv \frac{1}{N^{4}} \operatorname{Tr}\left(\left\{T_{\mathbf{A}}, T_{\mathbf{B}}\right\} T_{\mathbf{C}}\right)=2 \cos \frac{2 \pi(\mathbf{A} \times \mathbf{B})}{N} \delta_{\mathbf{A}+\mathbf{B}+\mathbf{C}} .
$$

In (A.18) we have extracted an extra factor $N$ in order to ensure that $\tilde{d}_{\mathbf{A B C}}$ remains finite in the limit $N \rightarrow \infty$. At this point, the advantage of choosing the phase factor as in (A.10) becomes clear: the tensors $\tilde{f}_{\mathbf{A B C}}$ and $\tilde{d}_{\mathbf{A B C}}$ now satisfy the proper periodicity requirements for shifts by $N$ unit lattice vectors. In the large- $N$ limit it is obvious that

$$
\begin{aligned}
& \tilde{f}_{\mathrm{ABC}}=-\frac{1}{\pi} f_{\mathrm{ABC}}+O\left(\frac{1}{N^{2}}\right), \\
& \tilde{d}_{\mathrm{ABC}}=2 d_{\mathrm{ABC}}+O\left(\frac{1}{N^{2}}\right) .
\end{aligned}
$$

Thus, the torus tensors $f_{\mathrm{ABC}}$ and $d_{\mathrm{ABC}}$ can be approximated by the $S U(N)$ tensors $\tilde{f}_{\mathbf{A B C}}$ and $\tilde{d}_{\mathbf{A B C}}$, and moreover the large- $N$ corrections can be determined explicitly. As an example, let us look at (2.39) in the finite- $N$ approximation. Substituting (A.17) and (A.18), we find

$$
\tilde{d}_{\mathrm{ABC}} \tilde{f}_{[\mathbf{D E}}^{\mathrm{A}} \tilde{f}_{\mathrm{F}] \mathrm{G}}^{\mathbf{B}}=O\left(\frac{1}{N^{2}}\right) \delta_{\mathbf{C}+\mathbf{D}+\mathbf{E}+\mathbf{F}+\mathbf{G}}
$$

so that (2.39) is only valid in the limit $N \rightarrow \infty$.

As already pointed out in Sect. 2, the definition of $c_{\mathbf{A B C}}$ for finite $N$ is ambiguous. We may, for instance, adopt the following definition,

$$
\tilde{c}_{\mathbf{A B C}}=\frac{2 \pi}{N} \sin \frac{2 \pi \mathbf{A} \cdot \mathbf{C}}{N}\left[2-\cos \frac{2 \pi A_{1}}{N}-\cos \frac{2 \pi A_{2}}{N}\right]^{-1} \delta_{\mathbf{A}+\mathbf{B}+\mathbf{C}},
$$

which is periodic and tends to (A.5) in the large- $N$ limit. Another question is 
whether, for finite $N$, one can also incorporate the structure constants $f_{\lambda \mathbf{A B}}$ associated with the vectors (A.6) by an extension of the $S U(N)$ algebra. However, imposing the Jacobi identity (2.46) for the case of $\mathbf{A}+\mathbf{B}+\mathbf{C}=\mathbf{0}$, we find that

$$
\tilde{f}_{\lambda \mathbf{A}(-\mathbf{A})}+\tilde{f}_{\lambda \mathbf{B}(-\mathbf{B})}+\tilde{f}_{\lambda(-\mathbf{A}-\mathbf{B})(\mathbf{A}+\mathbf{B})}=0 .
$$

Furthermore $\tilde{f}_{\lambda \mathbf{A B}}$ must be antisymmetric under the interchange of $\mathbf{A}$ and $\mathbf{B}$. Under these conditions we can show that there is no periodic solution to (A.23) so that no consistent extension of the $S U(N)$ structure constants exists that includes $\tilde{f}_{\lambda \mathbf{A B}}$. Nonetheless, at the level of the group, it is possible to include the corresponding finite transformations. For the infinite lattice, the transformations generated by (A.6) are simply the shifts $\sigma^{r} \rightarrow \sigma^{r}+c^{r}$, where $c^{r}$ is a constant vector. On the basis functions (A.1) this induces the transformation

$$
Y_{\mathbf{A}}(\sigma) \rightarrow \exp (i \mathbf{c} \cdot \mathbf{A}) Y_{\mathbf{A}}(\sigma) .
$$

For the finite lattice, the phase factors for finite shifts by $(4 i \pi / N) \mathbf{e}$, where $\mathbf{e}$ is a unit lattice vector, are obtained by conjugation with $\Omega_{1}$ and $\Omega_{2}$,

$$
\begin{aligned}
& \Omega_{1} T_{\mathrm{A}} \Omega_{1}^{-1}=\exp \left(\frac{4 \pi i A_{1}}{N}\right) T_{\mathrm{A}}, \\
& \Omega_{2}^{-1} T_{\mathrm{A}} \Omega_{2}=\exp \left(\frac{4 \pi i A_{2}}{N}\right) T_{\mathrm{A}} .
\end{aligned}
$$

\section{Appendix B: Area-Preserving Diffeomorphisms on $\boldsymbol{S}^{2}$}

Several discussions of area-preserving diffeomorphisms on $S^{2}$ have appeared in the literature $[3,7,9,11]$, which mainly deal with the structure constants and their $S U(N)$ approximation. In this appendix we present a uniform treatment of all the tensors that were introduced in Sect. 2. To facilitate their derivation we make use of a two-component $(S U(2))$ notation for the spherical harmonics.

In terms of the standard angular coordinates on $S^{2}, \sigma_{1}=\phi \in[0,2 \pi)$ and $\sigma_{2}=\theta \in[0, \pi)$, the induced metric for a two-dimensional sphere of unit area reads

$$
w_{r s}(\phi, \theta)=\frac{1}{4 \pi}\left(\begin{array}{cc}
\sin ^{2} \theta & 0 \\
0 & 1
\end{array}\right) .
$$

The basis functions $Y_{A}$ are now the spherical harmonics, which can be represented as symmetric traceless polynomials of a three-dimensional vector $\vec{X}(\phi, \theta)$ of unit length. It is convenient to adopt a two-component notation, where three-vectors are written as

$$
X^{\alpha \beta}=X^{\beta \alpha} \equiv X^{i}\left(\sigma_{2} \sigma_{i}\right)^{\alpha \beta},
$$

with $\alpha, \beta, \ldots=1,2$ and $\sigma_{i}$ the Pauli spin matrices. For a real vector $\vec{X}$ we have a corresponding reality condition

$$
X_{\alpha \beta} \equiv\left(X^{\alpha \beta}\right)^{*}=\varepsilon_{\alpha \gamma} \varepsilon_{\beta \delta} X^{\gamma \delta} .
$$

Consider now the set of functions $Y^{\alpha(2 n)}(\phi, \theta)$, where $\alpha(2 n)$ denotes an array of $2 n$ 
symmetrized indices $\alpha_{1}, \alpha_{2}, \ldots, \alpha_{2 n}$, which are defined as the symmetrized product of $n$ coordinates $X^{\alpha \alpha^{\prime}}(\phi, \theta)$,

$$
Y^{\alpha(2 n)} \equiv X^{\left(\alpha_{1} \alpha_{2}\right.} X^{\alpha_{3} \alpha_{4}} \cdots X^{\alpha_{\left.2 n-1 \alpha_{2 n}\right)}} .
$$

For given $n$ we have thus $2 n+1$ independent polynomials, which transform according to an irreducible representation of $S O(3)$. Observe that the $Y^{\alpha(2 n)}$ are not normalized,

$$
\int d \phi d \theta \sqrt{w(\phi, \theta)} Y^{\alpha(2 m)} Y^{\beta(2 n)}=\frac{\left(m ! 2^{m}\right)^{2}}{(2 m+1) !} \delta^{m n} \underbrace{\varepsilon^{\alpha \beta} \varepsilon^{\alpha \beta} \cdots \varepsilon^{\alpha \beta}}_{2 m},
$$

where symmetrization (with unit strength) over the indices in each array $\alpha(2 n)$, $\beta(2 n)$, etc., is understood henceforth. Furthermore, we have

$$
D^{r} D_{r} Y^{\alpha(2 n)}=-\vec{L}^{2} Y^{\alpha(2 n)}=-n(n+1) Y^{\alpha(2 n)},
$$

where $\vec{L}$ are the angular momentum operators, $L_{i}=-i \varepsilon_{i j k} X^{j} \partial / \partial X^{k}$, or, in two-component notation,

$$
L^{\alpha \beta}=2 i \varepsilon^{\gamma(\alpha} X^{\beta) \delta} \frac{\partial}{\partial X^{\gamma \delta}}
$$

Here $\partial / \partial X^{\alpha \beta} \equiv \frac{1}{2}\left(\sigma^{i} \sigma_{2}\right)^{\alpha \beta} \partial / \partial X^{i}$, so that

$$
\begin{aligned}
\frac{\partial}{\partial X^{\alpha \beta}} X^{\gamma \delta} & =\delta_{(\alpha}^{\gamma} \delta_{\beta)}^{\delta}, \\
{\left[L^{\alpha \beta}, L^{\gamma \delta}\right] } & =-2 i \varepsilon^{(\alpha(\gamma} L^{\delta) \beta)} .
\end{aligned}
$$

Any product of two functions $Y^{\alpha(2 m)}$ and $Y^{\beta(2 n)}$ can be decomposed into the same basis functions,

$$
Y^{\alpha(2 m)} Y^{\beta(2 n)}=\sum_{k} D_{k}^{m n} \underbrace{\varepsilon^{\alpha \beta} \varepsilon^{\alpha \beta} \cdots \varepsilon^{\alpha \beta}}_{m+n-k} Y^{\alpha(m-n+k) \beta(n-m+k)},
$$

where $k=m+n, m+n-2, \ldots,|m-n|$. The real tensor $D_{k}^{m n}$ is directly related to the tensor $d_{A B C}$, after the appropriate normalization factors for the basis functions $Y_{A}$ are taken into account, and satisfies the following relations,

$$
\begin{aligned}
D_{k}^{m n}=D_{k}^{n m}, \quad D_{m+n}^{m n} & =1, \quad D_{m-1}^{m 1}=\frac{2 m}{2 m+1}, \\
\frac{m+n-k+2}{m+n+k+1} D_{k-2}^{m n} & =\frac{k^{2}-(m-n)^{2}}{4 k^{2}-1} D_{k}^{m n}, \\
\frac{n-m+k}{2 n} D_{k}^{m n} & =\frac{2 k+1}{m+n+k+1} D_{k-1}^{m n-1} .
\end{aligned}
$$

The above equations are sufficient to determine $D_{k}^{m n}$. While (B.11) is still rather obvious, the proof of (B.12-13) is more involved and will be discussed later. After multiplication of (B.10) with another spherical harmonic and integration over $S^{2}$, 
one readily deduces that $\left(\left(k ! 2^{k}\right)^{2} /(2 k+1) !\right) D_{k}^{m n}$ must be symmetric in $m, n$ and $k$.

For the evaluation of the tensors $f_{A B C}$ and $c_{A B C}$ we first derive the following expressions for arbitrary functions $A$ and $B$ of $X^{\alpha \beta}(\phi, \theta)$,

$$
\begin{gathered}
\{A, B\}=4 \pi i \frac{\partial A}{\partial X^{\alpha \beta}}\left(L^{\alpha \beta} B\right)=-8 \pi X^{\alpha \beta} \varepsilon^{\gamma \delta} \frac{\partial A}{\partial X^{\alpha \gamma}} \frac{\partial B}{\partial X^{\beta \delta}}, \\
w^{r s} D_{r} A D_{s} B=-\frac{1}{2}\left(L^{\alpha \beta} A\right)\left(L_{\alpha \beta} B\right)=\left(X^{\alpha \beta} X^{\gamma \delta}-2 X^{\alpha \gamma} X^{\beta \delta}\right) \frac{\partial A}{\partial X^{\alpha \beta}} \frac{\partial B}{\partial X^{\gamma \delta}} .
\end{gathered}
$$

Choosing $A=Y^{\alpha(2 m)}$ and $B=Y^{\beta(2 n)}$ and decomposing into the same set of basis functions leads to two real tensors, $F_{k}^{m n}$ and $C_{k}^{m n}$, which are related to the tensors $f_{A B C}$ and $c_{A B C}$ after proper normalization,

$$
\begin{aligned}
\left\{Y^{\alpha(2 m)}, Y^{\beta(2 n)}\right\} & =\sum_{k} F_{k}^{m n} \underbrace{\varepsilon^{\alpha \beta} \varepsilon^{\alpha \beta} \cdots \varepsilon^{\alpha \beta}}_{m+n-k} Y^{\alpha(m-n+k) \beta(n-m+k),} \\
\frac{-2}{m(m+1)} D^{r} Y^{\alpha(2 m)} D_{r} Y^{\beta(2 n)} & =\sum_{k} C_{k}^{m n} \underbrace{\varepsilon^{\alpha \beta} \varepsilon^{\alpha \beta} \cdots \varepsilon^{\alpha \beta}}_{m+n-k} Y^{\alpha(m-n+k) \beta(n-m+k)},
\end{aligned}
$$

where

$$
\begin{aligned}
F_{k}^{m n} & =-8 \pi \frac{m n(2 k+1)}{m+n+k} D_{k-1}^{m-1 n-1}, \\
C_{k}^{m n} & =\frac{k(k+1)-m(m+1)-n(n+1)}{m(m+1)} D_{k}^{m n} .
\end{aligned}
$$

In deriving (B.18) and (B.19) we made use of (B.10) and (B.13). Note that $F_{k}^{m n}$ and $C_{k}^{m n}$ are zero whenever $m+n-k$ is even or odd, respectively. Obviously the Jacobi identity (2.7) for the Lie brackets gives rise to a corresponding identity for the tensor $F_{k}^{m n}$.

The structure constants can be approximated by those of $S U(N)$. To show this one introduces the matrices [3]

$$
T^{\alpha(2 n)} \equiv\left(\frac{4}{N^{2}-1}\right)^{(1 / 2)(n-1)} L^{\left(\alpha_{1} \alpha_{2}\right.} L^{\alpha_{3} \alpha_{4}} \cdots L^{\left.\alpha_{2 n-1} \alpha_{2 n}\right)},
$$

where the $L^{\alpha \beta}$ are the generators of $S O(3)$ in the $N$-dimensional representation, so that $L^{\alpha \beta} L_{\alpha \beta}=\frac{1}{2}\left(N^{2}-1\right)$. These matrices satisfy the following conditions:

$$
\begin{aligned}
T^{\alpha(2 n)} & =0 \quad \text { for } n \geqq N, \\
\left(T^{\alpha(2 n)}\right)^{\dagger} & =\varepsilon_{\alpha \beta} \cdots \varepsilon_{\alpha \beta} T^{\beta(2 n)}, \\
\operatorname{Tr} T^{\alpha(2 n)} & =0 \text { for } n \neq 0 .
\end{aligned}
$$

As all the $T^{\alpha(2 n)}$ are independent, there are $\sum_{n=1}^{N-1}(2 n+1)=N^{2}-1$ traceless independent matrices $T^{\alpha(2 n)}, 0<n<N$, which we may choose as the generators of $S U(N)$. If we also include the identity matrix, $T^{0}=\sqrt{\left(\left(N^{2}-1\right) / 4\right)} 1$, then these 
matrices close under multiplication,

$$
T^{\alpha(2 m)} T^{\beta(2 n)}=\sum_{k}\left\{\begin{array}{c}
m \\
k
\end{array}\right\} \underbrace{\varepsilon^{\alpha \beta} \varepsilon^{\alpha \beta} \cdots \varepsilon^{\alpha \beta}}_{m+n-k} T^{\alpha(m-n+k) \beta(n-m+k)},
$$

where $k=m+n, m+n-1, \ldots,|m-n|$. We note the following properties of the coefficients $\left\{\begin{array}{c}m \\ k\end{array}\right\}$,

$$
\begin{aligned}
& \left(\left\{\begin{array}{c}
m n \\
k
\end{array}\right\}\right)^{*}=(-)^{m+n-k}\left\{\begin{array}{c}
m n \\
k
\end{array}\right\} \\
& \left\{\begin{array}{c}
m n \\
k
\end{array}\right\}=\left\{\begin{array}{c}
n m \\
k
\end{array}\right\} \\
& \left\{\begin{array}{c}
m \quad n \\
m+n
\end{array}\right\}=\sqrt{\frac{N^{2}-1}{4}} \\
& \left\{\begin{array}{c}
m \\
m+n-1
\end{array}\right\}=-i m n \text {, } \\
& \left\{\begin{array}{c}
m \\
m-1
\end{array}\right\}=\frac{2 m}{2 m+1} \frac{N^{2}-m^{2}}{N^{2}-1} \sqrt{\frac{N^{2}-1}{4}}, \\
& \operatorname{Tr}\left(T^{\alpha(2 m)} T^{\beta(2 n)}\right)=\frac{\left(m ! 2^{m}\right)^{2}}{(2 m+1) !} \delta^{m n} \underbrace{\varepsilon^{\alpha \beta} \varepsilon^{\alpha \beta} \cdots \varepsilon^{\alpha \beta}}_{2 m}\left(\frac{N\left(N^{2}-1\right)}{4} \prod_{k=1}^{m} \frac{N^{2}-k^{2}}{N^{2}-1}\right) .
\end{aligned}
$$

From (B.30) we may define an $S U(N)$-invariant metric, which, up to normalization factors, yields the tensor $\eta_{A B}$ for the spherical harmonics in the $N \rightarrow \infty$ limit (cf. (B.5)).

If the matrices $L^{\alpha \beta}$ were commuting then $\left\{\begin{array}{c}m_{k} n \\ k^{n}\end{array}\right\}$ would coincide with $D_{k}^{m n}$, up to an overall factor $\sqrt{\left(N^{2}-1\right) / 4}$. The fact that the $L^{\alpha \beta}$ are not commuting gives rise to additional contributions, but those will be suppressed by powers of $\sqrt{4 /\left(N^{2}-1\right)}$. Using this observation and the intermediate result shown in (B.14), one can establish the following result,

$$
\left\{\begin{array}{c}
m n \\
k
\end{array}\right\}= \begin{cases}\sqrt{\frac{N^{2}-1}{4}}\left[D_{k}^{m n}+O\left(\frac{1}{N^{2}}\right)\right], & \text { for } m+n-k \text { even } \\
-\frac{i}{8 \pi}\left[F_{k}^{m n}+O\left(\frac{1}{N^{2}}\right)\right], & \text { for } m+n-k \text { odd }\end{cases}
$$

Observe that (B.31) is indeed consistent with various results that have been listed above. As far as the large- $N$ behaviour is concerned, there is a qualitative agreement between (B.31) and the coefficients that arise in the products of the $S U(N)$ generators for the torus (cf. (A.19-20)). The $S U(N)$ approximation to the tensors $f_{A B C}$ and $d_{A B C}$ is therefore given by the coefficients $\left\{\begin{array}{c}m_{k}{ }_{k} \\ \text { n }\end{array}\right\}$ and $\sqrt{4 /\left(N^{2}-1\right)}\left\{\begin{array}{c}m_{k}{ }_{k} \\ k\end{array}\right\}$, multiplied by the appropriate product of $\varepsilon$-symbols and the normalization factors for the spherical harmonics. The $S U(N)$ approximation of the tensor $c_{A B C}$ then follows from (B.19).

The coefficients $\left\{\begin{array}{cc}m & n \\ k\end{array}\right\}$ are subject to the following recurrency relations, which, 
when combined with (B.26-28), allow their complete determination,

$$
\begin{aligned}
& \frac{m+n-k+2}{m+n+k+1}\left\{\begin{array}{c}
m \\
k-2
\end{array}\right\}-\frac{k^{2}-(m-n)^{2}}{4 k^{2}-1} \frac{N^{2}-k^{2}}{N^{2}-1}\left\{\begin{array}{c}
m \\
k
\end{array}\right\} \\
& =-\frac{i}{\sqrt{N^{2}-1}} \frac{k(k-1)-m(m+1)-n(n+1)}{m+n+k+1}\left\{\begin{array}{cc}
m & n \\
k-1
\end{array}\right\}, \\
& \frac{n-m+k}{2 n}\left\{\begin{array}{c}
m n \\
k
\end{array}\right\}-\frac{2 k+1}{m+n+k+1}\left\{\begin{array}{cc}
m & n-1 \\
k-1
\end{array}\right\} \\
& =-\frac{i}{\sqrt{N^{2}-1}} \frac{k(k+1)-n(n-1)+m(n+k)}{m+n+k+1}\left\{\begin{array}{c}
m n-1 \\
k
\end{array}\right\}, \\
& \frac{m+n-k}{2 n}\left\{\begin{array}{c}
m n \\
k
\end{array}\right\}-\frac{m-n+k+2}{2 k+3} \frac{N^{2}-n^{2}}{N^{2}-1}\left\{\begin{array}{cc}
m \\
k+1
\end{array}\right\} \\
& =-\frac{i}{\sqrt{N^{2}-1}} \frac{(m+n+k+2)(m-n-k-1)(n-k-1)}{2 n(2 k+3)}\left\{\begin{array}{cc}
m & n \\
k+1
\end{array}\right\} .
\end{aligned}
$$

These relations have been derived by using the associativity of the matrix product, $\left(T^{\alpha(2 m)} T^{\beta(2 n)}\right) T^{\gamma(2)}=T^{\alpha(2 m)}\left(T^{\beta(2 n)} T^{\gamma(2)}\right)$, and substituting (B.24) and the explicit expressions for $\left\{\begin{array}{c}m_{k} \\ k\end{array}\right\}$. The latter have been calculated separately (cf. (B.27-29)). For $m+n-k$ even, (B.32-34) become degenerate in the large- $N$ limit and yield (B.12-13). Obviously, the associativity of the matrix product can be used to derive more identities for products of the $\left\{\begin{array}{c}m \\ k\end{array}\right\}$.

Finally, we come to the relation between the area-preserving diffeomorphisms generated in the $N \rightarrow \infty$ limit of the torus and the sphere. Clearly, for finite $N$, the generators $T_{\mathrm{A}}$ of the torus algebra can be decomposed in terms of the generators $T^{\alpha(2 n)}$ of the sphere algebra. For instance, using the results given above, we can explicitly compare the diagonal generators of both algebras. In a basis where $L^{12}$ (which is equal to $i L^{3}$ in Cartesian coordinates) is diagonal, we may choose $\Omega_{1}=\exp \left(4 \pi L^{12} / N\right)$, so that

$$
T_{(A, 0)}=\sqrt{\frac{4 N^{2}}{N^{2}-1}} \sum_{n=0}^{N-1} M_{n 0} T^{1(n) 2(n)},
$$

where $M_{n 0}$ are the elements of an $N \times N$ matrix $M$, defined by

$$
\begin{aligned}
& (\ln M)_{n-1 n}=-2 \pi A \frac{n^{2}}{4 n^{2}-1} \frac{N^{2}-n^{2}}{N \sqrt{N^{2}-1}}, \\
& (\ln M)_{n n-1}=2 \pi A \frac{\sqrt{N^{2}-1}}{N}, \quad n=0, \ldots, N-1,
\end{aligned}
$$

and all remaining elements of $\ln M$ equal to zero. From the left-hand side of (B.35) it is clear that $M_{00}=0$, unless $A=0 \bmod N$, although this property is not manifest for the right-hand side of this equation. For $N \rightarrow \infty$, (B.35) defines a map between the torus functions $Y_{(A, 0)}$ and functions on the sphere, expressed as a series 
expansion in terms of the spherical harmonics $Y^{1(n) 2(n)}$. Although the functions corresponding to (B.35) are relatively simple, as they are only functions of one coordinate of the torus and the sphere, respectively, this example demonstrates the complexity of the relation between the two sets of functions. However, in view of the different topological structure of the torus and the sphere, it is unlikely that the torus functions $Y_{\mathbf{A}}$ will always lead to functions that are differentiable on the sphere.

Acknowledgements. We are grateful to E. Bergshoeff and M. Lüscher for clarifying discussions.

Note added. Recently, the relation between the algebra of area-preserving diffeomorphisms on $S^{2}$ and the algebras used for the description of higher-spin gauge fields [22] received some attention $[11,23]$.

\section{References}

1. Bergshoeff, E., Sezgin, E., Townsend, P. K.: Ann. Phys. 185, 330 (1988); Townsend, P. K.: In: Superstrings '88'. Proc. of the Trieste Spring School. Green, M. B., Grisaru, M. T., Iengo, R., Strominger, A. (eds.). Singapore: World Scientific 1989; Duff, M. J.: Class. Quant. Grav. 5, 189 (1988)

2. Goldstone, J.: unpublished

3. Hoppe, J.: MIT PhD Thesis, 1982, and in Proc. Int. Workshop on Constraints Theory and Relativistic Dynamics. Longhi, G., Lusannna, L. (eds.), Singapore: World Scientific 1987

4. Arnold, V. I.: Mathematical methods of classical mechanics, Berlin, Heidelberg, New York: Springer 1978

5. Bergshoeff, E., Sezgin, E., Townsend, P. K.: Phys. Lett. 189B, 75 (1987)

6. Floratos E., Iliopoulos, J.: Phys. Lett. 201B, 237 (1988)

7. de Wit, B., Hoppe, J., Nicolai, H.: Nucl. Phys. B305 [FS23], 545 (1988)

8. Bars, I., Pope, C. N., Sezgin, E.: ICTP preprint IC/88/77

9. Arakelyan, T. A., Savvidy, G. K.: Phys. Lett. B214, 350(1988), preprint FERMILAB-PUB-88/203-T

10. Fairlie, D. B., Fletcher, P., Zachos, C. K.: preprint ANL-HEP-PR-88-56, DTP-88-13

11. Bergshoeff, E., Blencowe, M. P., Stelle, K. S.: Imperial College preprint TH 88-89-9 (1988)

12. Hoppe, J.: Karlsruhe preprint KA-THEP-18 (1988)

13. Banyaga, A.: Commun. Math. Helv. 53, 174 (1978)

14. de Wit, B., Lüscher, M., Nicolai, H.: Nucl. Phys. B320, 135 (1989)

15. Marquard, U., Scholl, M.: Phys. Lett. B209, 434 (1988); Phys. Lett. 227B, 227 (1989)

16. Kaiser, R., Marquard, U., Scholl, M.: Phys. Lett. 227B, 234 (1989)

17. de Wit, B., Hull, C. M., Roček, M.: Phys. Lett. 184B, 233 (1987)

18. Dirac, P. A. M.: Lectures on quantum mechanics, London: Academic Press 1967

19. Lüscher, M.: private communication

20. 't Hooft, G.: Commun. Math. Phys. 81, 267 (1981)

21. van Baal, P.: Commun. Math. Phys. 92, 1 (1983); Fabricius, K., Korthals-Altes, C. P.: Nucl. Phys. B240, 237 (1984); Lüscher, M., Weisz, P.: Nucl. Phys. B266, 309 (1986)

22. Fredkin, E. S., Vasiliev, M. A.: Ann. Phys. 177, 63 (1987); Konstein, S. E., Vasiliev, M. A.: Lebidev preprint 58 (1989) and references therein

23. Bordemann, M., Hoppe, J., Schaller, P.: Karlsruhe preprint KA-THEP-10-1989

Communicated by L. Alvarez-Gaumé

Received March 16, 1989 